\title{
Trop vieille pour avoir un bébé?
}

\section{Valérie Junod}

Professeure de droit aux Universités de Genève et de Lausanne

Le 10 mai dernier, le Tribunal fédéral a rendu un arrêt passé largement inaperçu, mais néanmoins intéressant [1]. Une patiente exigeait que sa caisse rembourse son (troisième) traitement d'infertilité consistant en une stimulation ovarienne suivie d'une insémination intra-utérine (IIU) $)^{1}$. Le traitement est normalement à charge des caisses, l'ordonnance sur les prestations dans l'assurance-maladie (OPAS) prévoyant explicitement le remboursement d'un maximum de trois cycles d'IIU (par grossesse). Malgré cette mention explicite, la caisse refusait le remboursement au motif que la patiente était alors âgée de 44 ans; pour la caisse, le traitement était dans son cas inefficace, car son infertilité était due à lâge, de sorte que la stimulation ovarienne suivie de l'IIU ne parviendrait pas (plus) à y remédier. L'arrêt mérite mention car le Tribunal fédéral est amené à prendre position sur le critère de l'âge invoqué pour exclure la prise en charge par l'assurance-maladie obligatoire. L'arrêt commence par rappeler que pour être remboursé, une prestation doit viser le traitement d'une maladie $^{2}$ et qu'elle doit être, à ce titre, efficace, appropriée et économique. C'est ce premier critère d'efficacité qui donnait lieu à une divergence de vues entre la caisse et l'assurée. Sur le principe, la mise en œuvre de ce critère était rendue plus facile, dès lors que l'IIU est - comme déjà relevé - explicitement inscrite dans le catalogue des prestations à rembourser. Le DFI (Département fédéral de l'intérieur), l'OFSP (Office fédéral de la santé publique) et sa commission spécialisée se sont déjà prononcés (positivement) sur son efficacité. Le Tribunal fédéral a pour habitude de ne pas remettre en cause leur appréciation, dès lors qu'elle figure dans l'annexe 1 de l'OPAS.

\section{Pathologie et âge: deux raisons d'infer- tilité qu'il faut différencier}

Cependant, dans cette affaire, le Tribunal fédéral considère qu'il faut différencier entre infertilité due à une pathologie et celle due à l'âge: «les mesures médicales visant l'amélioration de la capacité à procréer en cas de baisse de la fertilité liée exclusivement à l'âge ne constituent pas le traitement d'une maladie»³. Dans pareille situation, ces mesures n'entrent pas dans le champ des prestations à charge des caisses selon la LAMal.

\section{Zu alt, um ein Kind zu bekommen?}

Die Krankenversicherungskosten steigen unaufhörlich an, und die Rationierung von medizinischen Leistungen ist nach wie vor umstritten. In seinem Entscheid vom 10. Mai 2016 musste sich das Bundesgericht zu einer allfälligen Altersgrenze äussern, über der eine Behandlung - im vorliegenden Fall eine intrauterine Insemination - nicht mehr wirksam und wirtschaftlich ist. Dabei handelt es sich um eine heikle Frage, da sie mit medizinischen, wirtschaftlichen, sozialen und ethischen Aspekten verbunden ist. Das Bundesgericht nimmt diesbezüglich eine vorsichtige Haltung ein, so dass die Verantwortung für den Entscheid, ob die medizinische Leistung erbracht werden soll, hauptsächlich bei der Ärzteschaft liegt. In diesem Beitrag werden die Schwierigkeiten in der Praxis dargelegt, die mit einer solchen Lösung verbunden sind.

Cependant, la difficulté juridique découle de la difficulté sur le plan médical à attribuer une cause à l'infertilité. Il n'existe pas de test diagnostique permettant d'attribuer une cause certaine à un trouble de la fertilité, en particulier un test permettant de l'imputer «exclusivement à l'âge». Comme le relève le Tribunal:

On ignore quel serait le taux de succès si bas que le traitement devrait être qualifié de (juridiquement) inefficace.

«Une limite d'âge à partir de laquelle une femme ne pourrait plus tomber enceinte ni mener une grossesse à terme n'a [...] pas été arrêtée.» Si l'on doit estimer une période limite, elle varie «entre 42 et 51 ans». Vu notamment l'ampleur de cette fourchette, la LAMal ne fixe aucune limite d'âge pour le remboursement. La Loi sur la procréation médicalement assistée (LPMA) n'en fixe pas non plus, même si elle exige que les parents soient suffisamment jeunes pour pouvoir élever leur futur enfant jusqu'à sa majorité. Ce choix du législateur, comme le souligne le Tribunal fédéral, était délibéré, toute fixation de limite étant de nature à engendrer des problèmes (par ex. des tentatives des patientes poursuivies jusqu'au seuil limite, des refus systématiques des caisses pour un dépassement, même minime, de l'âge limite). La ménopause n’a pas davantage été retenue comme un critère susceptible d'être inscrit comme seuil dans la LPMA, ne serait-ce qu'en raison de l'inégalité biologique entre les femmes, certaines l'atteignant beaucoup plus tôt que d'autres. Le Tribunal écarte 


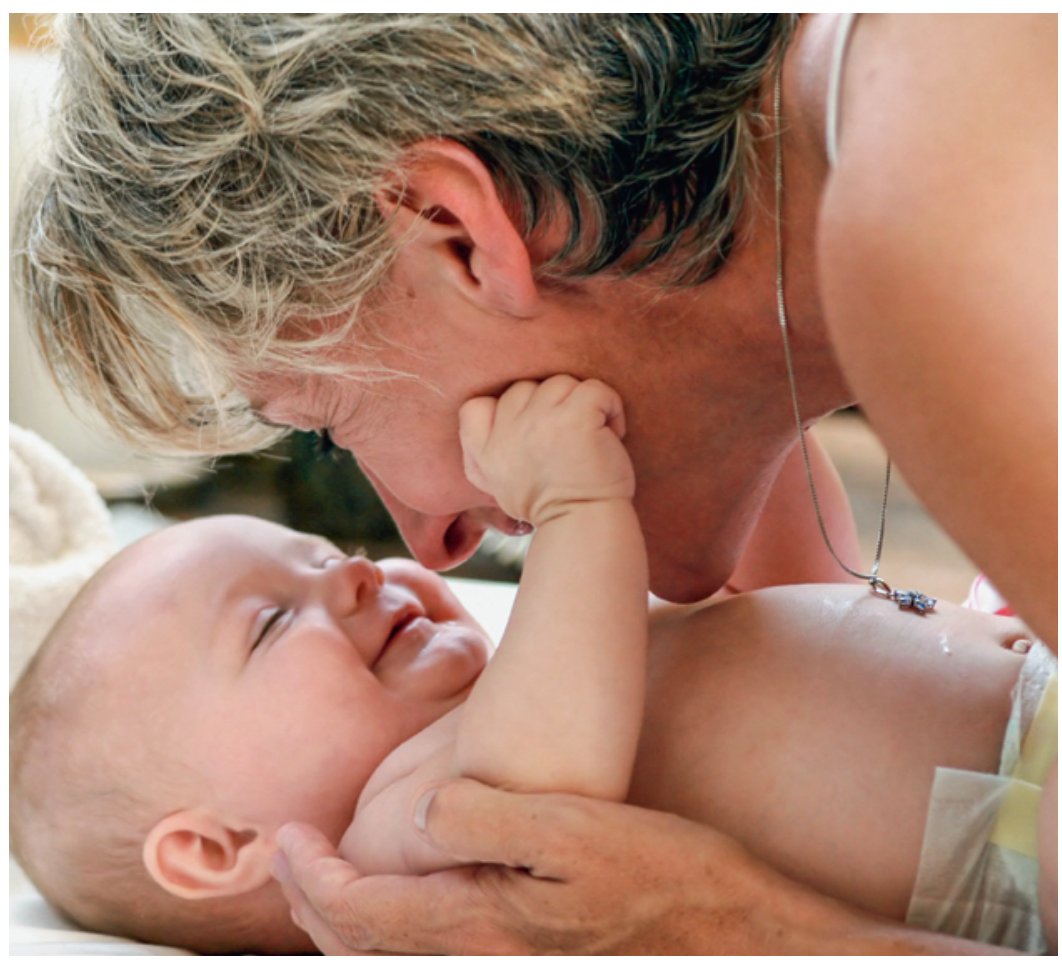

Jusqu'à quel âge accepte-t-on que les femmes donnent naissance? La question est délicate car elle présente des enjeux à la fois médicaux, juridiques, économiques, sociaux et éthiques.

encore le manuel de la Société suisse des médecinsconseils et des médecins d'assurance (SSMC) selon lequel 40 ans serait l'âge limite pour un remboursement; il estime que ce seuil a «été adopt[é] par une association de médecins-conseils et de médecins d'assurance» sans fournir «les raisons de la fixation d'une telle limite». Enfin, il juge que la baisse chez la patiente du (seul) taux d'hormone antimüllerienne ne suffit pas à retenir l'inefficacité du traitement.

De cette analyse, le Tribunal fédéral conclut que le «seul critère de l'âge ne saurait en soi justifier la négation du caractère efficace du traitement». Il refuse de fixer lui-même un âge limite. Au contraire, il recommande «une approche individualisée fondée sur les composantes cliniques propres à chaque patiente».

Dans cette étape suivante de l'analyse, le Tribunal constate que le dossier de la patiente est insuffisant pour «apprécier [ses] chances de succès du traitement». Il aurait fallu disposer des données d'analyse biologique récentes (au regard de la date du traitement); ensuite, il aurait fallu «le cas échéant avec l'aide d'un expert médical» interpréter ces résultats d'analyse pour se prononcer sur l'efficacité anticipée du traitement. L'avis optimiste de la doctoresse qui suivait la patiente ne constitue pas une preuve satisfaisante. Le dossier étant incomplet, le Tribunal fédéral renvoie la cause au tribunal cantonal pour que celui-ci ordonne les compléments nécessaires (par rap- port au traitement déjà intervenu en 2012). Le tribunal cantonal devra obtenir le dossier médical complet de la patiente, puis le soumettre à un expert spécialisé et indépendant.

Point important, le Tribunal fédéral juge que, si le dossier devait demeurer incomplet, notamment parce que les résultats d'analyses pour la période en cause (2012) ne sont pas ou plus disponibles, l'assurée en supporterait les conséquences; autrement dit, la caisse serait légitimée à lui refuser le remboursement. Le Tribunal justifie cette répartition du fardeau de la preuve au motif que la caisse avait déjà signalé sa réticence à rembourser un premier traitement (ce qu'elle avait pourtant fini par faire), de sorte que l'assurée «aurait dû être consciente de la nécessité de pouvoir disposer des valeurs actuelles rendant vraisemblable le fait que son infertilité constituait une maladie».

\section{Que faut-il retenir de cet arrêt?}

D'abord le problème de l'incertitude juridique. La LAMal opère avec un certain schématisme destiné notamment à garantir la sécurité juridique. En principe, les prestations ordonnées par les médecins sont remboursées. Celles qui sont nouvelles ou controversées sont inscrites dans l'OPAS, avec une brève description de leur statut (remboursées, non remboursées, remboursées à certaines conditions). Ici, l'IIU était inscrite dans l'OPAS avec une condition - en l'occurrence remplie. L'arrêt du Tribunal fédéral vient toutefois rappeler que cela ne suffit pas et que même une prestation à charge selon l'OPAS peut être refusée si, dans le cas concret, elle apparaît inefficace. Ceci constitue une brèche dans la sécurité juridique, puisque les assurés et les professionnels de la santé ne peuvent pas se fier entièrement à l'OPAS, mais doivent anticiper une possible appréciation au cas par cas.

Ensuite le problème de la fertilité. Le Tribunal fédéral doit admettre qu'il s'agit d'un domaine où les certitudes médicales font défaut. Identifier la cause d'une infertilité, même chez une patiente ayant atteint la quarantaine, est difficile. Malheureusement, la

\section{Le fait qu'ici l'âge faisait apparaître un} phénomène physiologique - l'infertilité comme naturel n'enlève rien aux difficultés.

deuxième partie de l'arrêt esquive cette difficulté, en donnant à penser que des résultats de tests (notamment des taux d'hormones) permettraient ensuite un diagnostic se prêtant à un jugement tranché en faveur ou contre le remboursement. Les tribunaux risquent d'être déçus: même avec une batterie de test, il reste 
très difficile de prédire si un traitement par stimulation suivi d'une IIU a des chances de succès. Le Tribunal s'est d'ailleurs abstenu de définir le seuil qui permettrait d'établir à l'inefficacité du traitement (10\% de naissance par cycle? 20\%? 30\%?). On ignore quel serait le taux de succès si bas que le traitement devrait être qualifié de (juridiquement) inefficace. On sait d'ailleurs que, par cycle, les chances de donner naissance à un enfant sont globalement bien inférieures à 50\% - ce qui n'a pas empêché l'OFSP, il y a de cela déjà bien des années, d'admettre l'efficacité de l'IIU.

Enfin, le problème de l'âge. Invoquer l'âge pour refuser le remboursement d'une prestation est toujours délicat. Le fait qu'ici l'âge faisait apparaître un phénomène

\section{Comment les caisses sont-elles censées procéder?}

physiologique - l'infertilité - comme naturel n'enlève rien aux difficultés. Il y a plusieurs autres troubles médicaux qui sont liés à l'âge. On peut considérer que la perte auditive, la perte d'acuité visuelle, la démence, la fragilité osseuse, les difficultés ambulatoires et respiratoires deviennent toutes «naturelles» à partir d'un certain âge. Or ce n'est pas parce que, avec l'âge, le trouble est devenu normal ou attendu qu'il ne faudrait pas (plus) le traiter. La question est plutôt de savoir s'il est efficace et économique de le traiter, lorsque les moyens médicaux existent a priori. Dans l'arrêt du Tribunal fédéral, les deux questions sont malheureusement traitées conjointement. Tantôt il est dit que l'infertilité ne serait plus une maladie chez la patiente d'un certain âge, tantôt il est expliqué que le traitement ne serait plus efficace. A notre avis, il serait prudent de concentrer l'analyse sur le deuxième aspect, en laissant de côté la question d'une infertilité devenue naturelle avec l'âge. En effet, cette question est avant tout socio-éthique: jusqu'à quel âge accepte-t-on que les femmes (voire les hommes pour d'autres traitements de l'infertilité) donnent naissance? A supposer qu'il soit vraiment nécessaire de donner une réponse à cette question, il serait alors préférable que celle-ci soit inscrite dans la loi. La faire trancher indirectement par un médecin-conseil d'une caisse n'est pas approprié. Plus généralement, la question de l'âge maximum pour le remboursement de prestations médicales mérite d'être examinée de manière plus globale. Comme déjà signalé, il existe une multitude de traitements dont l'efficacité baisse avec l'âge du patient. Comment les caisses sont-elles censées procéder? Dire, comme le fait le Tribunal fédéral, qu'il faut un examen individualisé de chaque cas n'apporte qu'un début de réponse. La question est en effet bien plus compliquée: elle soulève de larges enjeux d'équité, de justice, de solidarité, d'attitude face à la mort et à la vieillesse pour n'en citer que quelques-uns. Il est illusoire de penser pouvoir la trancher uniquement en fonction de critères médicaux.

\section{Crédit photo}

(c) Gerbera1 | Dreamstime.com

\section{Référence}

1 Arrêt 9C_435/2015 prévu pour publication dans la collection officielle du Tribunal fédéral; voir aussi le communiqué de presse du Tribunal sous http://www.bger.ch/fr/press-news-9c_435_2015-t.pdf. 